\title{
OXIMETRIA dO ESTÔMAGO: estudo COMPARATIVO ENTRE VAGOTOMIA GÁSTRICA PROXIMAL E SEROMIOTOMIA DA CURVATURA GÁSTRICA MENOR ANTERIOR COM VAGOTOMIA TRONCULAR POSTERIOR NA ÚLCERA DUODENAL CRÔNICA
}

* Carlos Haruo Arasaki, Júlio César Martinez, José Carlos Del Grande

Disciplina de Gastroenterologia Cirúrgica da Universidade Federal de São Paulo - Escola Paulista de Medicina (UNIFESP/EPM)

RESUMO - Retardo do esvaziamento gástrico de alimentos sólidos ocorre mais intensamente depois de vagotomia gástrica proximal (VGP) que após seromiotomia da curvatura gástrica menor anterior com vagotomia troncular posterior (SMAVTP), podendo estar relacionado à hipóxia da parede gástrica e, principalmente, do marcapasso gástrico.

OвJEtivo. Determinar a repercussão da desvascularização cirúrgica da curvatura menor e do fundo gástrico no estômago como um todo e, particularmente, na região do marcapasso.

Métodos. Mediu-se a saturação de oxigênio da hemoglobina $\left(\mathrm{SpO}_{2}\right)$ intra-operatória, por oximetria de pulso, da parede gástrica anterior em 20 portadores de úlcera duodenal crônica, alocados casualmente em dois grupos de 10 pacientes para tratamento cirúrgico por VGP ou SMAVTP.

Resultados. As medições, obtidas antes de fundoplicatura parcial, mostraram que o fundo gástrico e a curvatura menor proximal tiveram redução significante da $\mathrm{SpO}_{2}$ quando comparados ao corpo gástrico $(\mathrm{p}<0,05)$; que a $\mathrm{SpO}_{2}$, na região do marcapasso gástrico, não sofreu alteração estatisticamente significante; que a associação entre ligadura dos vasos gástricos curtos e VGP ou SMAVTP reduziu significantemente a $\mathrm{SpO}_{2}$ $(p<0,05)$; e que a técnica da VGP resultou em $\mathrm{SpO}_{2}$ significantemente menor que a SMAVTP $(p<0,05)$.

ConcLusão. A VGP, quando associada à ligadura dos vasos gástricos curtos, produz alterações isquêmicas agudas da parede gástrica, na região da curvatura menor proximal e do fundo gástrico, mais intensas que a SMAVTP. A região do marcapasso gástrico não sofre hipóxia imediatamente após os procedimentos operatórios.

UNITERMOS: Estômago. Úlcera duodenal. Vagotomia gástrica proximal. Oximetria. Relógios biológicos.

\section{INTRODUÇÃO}

A partir de 1969, a vagotomia gástrica proximal (VGP) começou a ser praticada amplamente no tratamento cirúrgico da úlcera duodenal. A seromiotomia da curvatura gástrica menor anterior com vagotomia troncular posterior (SMAVTP) foi introduzida, posteriormente, com premissa de facilitar a desnervação das células parietais, sem comprometer resultados benéficos do método.

Embora riscos de perfuração ou de necrose gástrica sejam maiores na VGP do que naSMAVTP,' acredita-se que as duas técnicas sejam equivalentes quanto à redução da secreção ácida e à taxa de recidiva ulcerosa ${ }^{2}$, e que aVGP mantém inalterado o esvaziamento gástrico de alimentos sólidos, pois a inervação

\footnotetext{
*Correspondência:

R. Dr. Diogo de Faria, 1087 conj. 308-V. Clementino CEP 04037-003-São Paulo-SP Tel./Fax:(011)5549-7658 e-mail:haruo@osite.com.br
}

motora antral estaria preservada ${ }^{3}$. Todavia, retardo do esvaziamento gástrico para sólidos, ainda que transitório, tem sido constatado, principalmente depois de VGP4,5, podendo a SMAVTP apresentar igual alteração de esvaziamento ${ }^{6}$ ou ser, nesse aspecto, mais eficiente que a VGP?.

Estudo prévio, indicando que a hipóxia pode comprometer a atividade mioelétrica coordenada pelo marcapasso gástri$\operatorname{co}^{8} \mathrm{e}$, dessa forma, resultar em distúrbios da motilidade desse órgão, explicaria as alterações de esvaziamento gástrico citadas anteriormente. Entretanto, a desvascularização do estômago na região do marcapasso, após a VGP e a SMAVTP, não foi ainda comprovada.

No intuito de se confirmar ou não a ocorrência de hipóxia tecidual na parede do estômago in vivo, particularmente nas proximidades do marcapasso gástrico, e se essa alteração relaciona-se com a técnica operatória empregada, realizou-se estudo comparativo entre as técnicas da VGP e da SMAVTP.

\section{Métodos}

\section{Seleção de pacientes}

Foram selecionados 20 doentes com úlcera duodenal crônica que permanecia em atividade, mesmo após tratamento com omeprazol ( $20 \mathrm{a} 40 \mathrm{mg}$ por dia) e amoxicilina ( 2 g por dia), ambos durante 14 dias, e com ranitidina ( $300 \mathrm{a} 600 \mathrm{mg}$ por dia) nas quatro semanas seguintes. Tais pacientes foram operados entre Agosto de 1995 a Junho de 1996.

\section{Randomização}

Dois grupos, com dez pacientes, foram formados mediante sorteio realizado no préoperatório. O exame da amostra estudada não revelou diferença significante entre os dois grupos em termos de distribuição por sexo e idade.

\section{Oxímetro de pulso}

O instrumento utilizado na medida da saturação de oxigênio foi um oxímetro de pulso (Ohmeda ${ }^{\circledR}$, modelo Biox 3700 , de Louisville, Colorado, E.U.A.), cujo sensor (EarProbe ${ }^{\circledR}$ ), 
TABELA I - Análise de variância de $\log (\mathrm{SpO})$ nos tempos inicial, pré-procedimento e pós-procedimento, medidos em seis pontos da parede anterior do estômago, em pacientes submetidos a cirurgia pela técnica da VGP ou da SMAVTP

\begin{tabular}{|c|c|c|c|}
\hline FontesdeVariação & GL & F calculado & $p$ \\
\hline EntrePontos & 5 & 8,407 & 0,000 \\
\hline Entre Tempos & 2 & 3,277 & 0,039 \\
\hline Entre Técnicas & I & 4,641 & 0,032 \\
\hline \multicolumn{4}{|l|}{ Interaçóes(Ia ordem) } \\
\hline Pontos $x$ Tempos & 10 & 0,934 & NS \\
\hline Pontos $x$ Técnicas & 5 & 0,549 & NS \\
\hline $\begin{array}{l}\text { Tempos x Técnicas } \\
\text { Interacóes (2 } 2^{\mathrm{a}} \text { ordem) }\end{array}$ & 2 & I, 127 & NS \\
\hline Pontos $x$ Tempos $x$ Técnicas & 10 & 0,750 & NS \\
\hline Resto & 324 & - & - \\
\hline Total & 359 & - & - \\
\hline
\end{tabular}

GL:Grausde Liberdade;NS:NãoSignificante

Gráfico I - Médias de log $(\mathrm{SpO})$ obtidas a partir de seis pontos da parede anterior do estômago de pacientes submetidos a cirurgia pela técnica da VGP ou da SMAVTP; as probabilidades calculadas referem-se ao Teste de Tukey entre Pontos

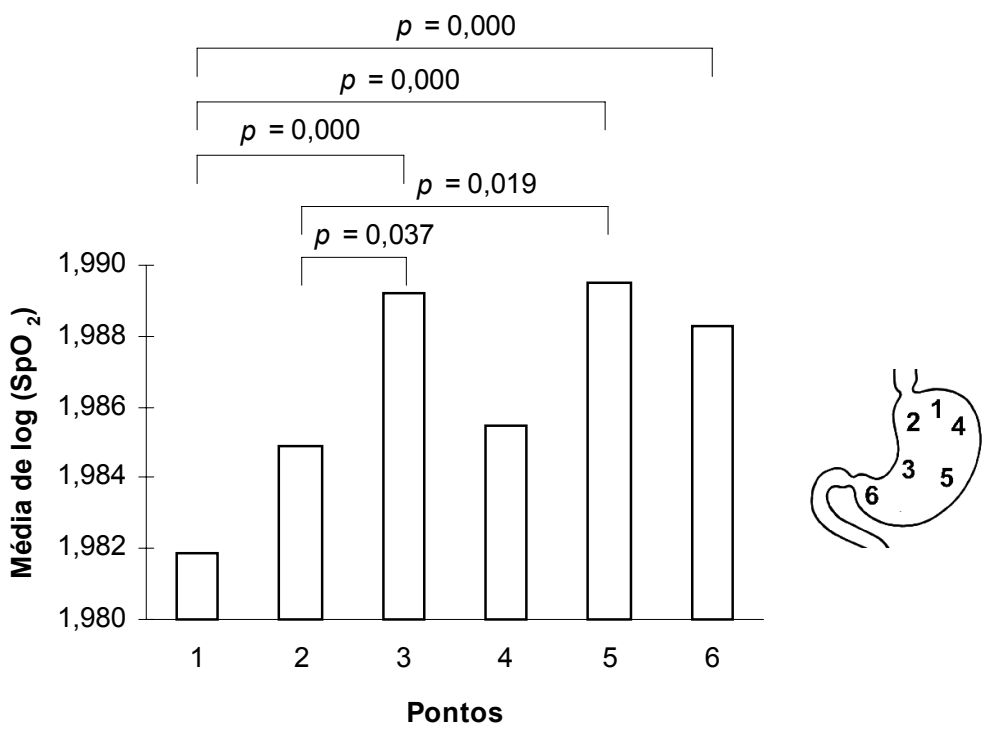

previamente esterilizado em óxido de etileno, foi acoplado a uma dobra da parede gástrica anterior, formada com auxílio de uma sonda nasogástrica posicionada na luz gástrica. Cada medida foi anotada somente 30 segundos após a instalação do sensor no estômago, e desde que acompanhada de onda pletismográfica com freqüência cardíaca fidedigna.

\section{Procedimentos operatórios}

Os pacientes foram submetidos à anestesia geral. Após a laparotomia, pontos de medição de oximetria foram demarcados pela aplicação de pontos frouxos temporários de fio de algodão nos seguintes locais:

I. Fundo gástrico: cerca de $3 \mathrm{~cm}$ à esquerda da incisura cárdica;

2. Parte proximal da curvatura gástrica menor: aproximadamente $2 \mathrm{~cm}$ distal à cárdia;

3. Parte distal da curvatura gástrica menor: ao nível da incisura angular;

4. Parte proximal da curvatura gástrica maior: ao nível do 3ำ vaso gástrico curto, contado a partir da incisura cárdica;

5. Parte média da curvatura gástrica maior: aproximadamente no ponto médio da curvatura maior;

6. Parte pilórica do estômago (antro): aproximadamente no ponto médio entre a curvatura gástrica maior e a menor e cerca de $3 \mathrm{~cm}$ aquém do piloro gastroduodenal.

Foram obtidas medidas de saturação de oxigênio da hemoglobina $\left(\mathrm{SpO}_{2}\right)$ inicial da parede gástrica anterior nas seis posições previamente demarcadas. A fim de se liberar o fundo gástrico, parcialmente realizou-se ligadura e secção de três vasos gástrico curtos proximais e de eventual vaso gástrico posterior. Procedeu-se, então, às medidas de $\mathrm{SpO}_{2}$ pré-VGP e pré-SMAVTP nos mesmos locais. As técnicas da VGP e da SMAVTP foram executadas e, posteriormente, mediuse a $\mathrm{SpO}_{2}$ pós-VGP e pós-SMAVTP naqueles seis pontos. Nos dois grupos, as operações foram finalizadas por meio de aproximação dos fascículos direito e esquerdo do pilar diafragmático direito, e de fundoplicatura parcial do esôfago, de $270^{\circ}$.

\section{Ética}

O protocolo do presente estudo foi aprovado pela Comissão de Ética Médica do Hospital São Paulo/Universidade Federal de São Paulo.

\section{Método estatístico}

Após a transformação logarítmica dos valores de $\mathrm{SpO}_{2}$, indicada pela expressão "log $\left(\mathrm{SpO}_{2}\right)$ ", utilizou-se a Análise de Variância a Três Níveis de Classificação na comparação entre três fatores - técnica operatória, local de medição da $\mathrm{SpO}_{2}$ e tempo de medição. $\mathrm{O}$ teste de comparaçôes múltiplas de Tukey foi aplicado, como complemento, sempre que houve significância estatística na Análise de Variância. Em todos os testes fixou-se em 0,05 ou $5 \%$ o nível de significância estatística.

\section{Resultados}

Ao todo, foram obtidas 360 medidas intraoperatórias de $\mathrm{SpO}_{2}$, sendo os logaritmos destes valores submetidos à Análise de Variância (Tabela I). As diferenças significantes obtidas são analisadas, a seguir, por meio de gráficos de médias de $\log \left(\mathrm{SpO}_{2}\right)$. Desse modo, os Gráficos I, 2 e 3 comparam as médias de log 
Gráfico 2 - Médias de $\log \left(S_{p} O_{2}\right)$ obtidas nos tempos inicial, pré-procedimento (PRÉ) e pós-procedimento (PÓS), na parede anterior do estômago de pacientes submetidos a cirurgia pela técnica da VGP ou da SMAVTP; a probabilidade calculada refere-se ao Teste de Tukey entre Tempos

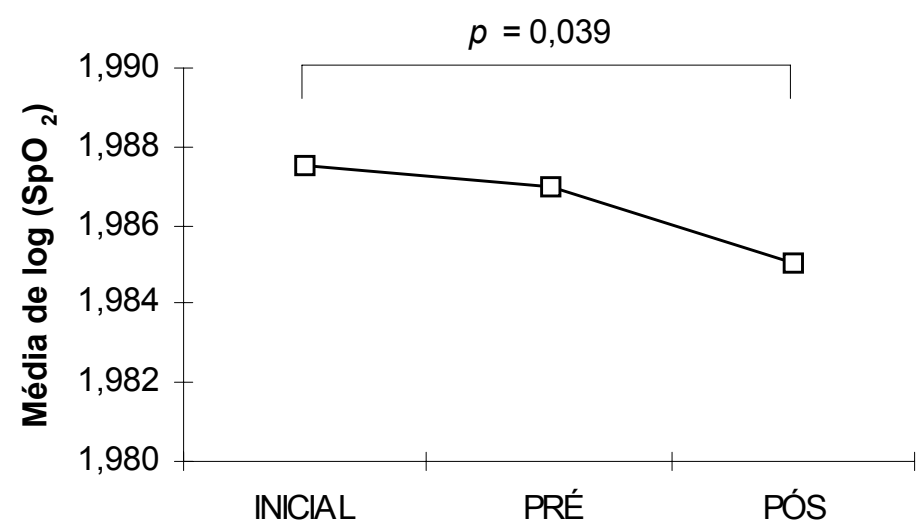

Tempos

Gráfico 3 - Médias de $\log \left(\mathrm{SpO}_{2}\right)$ obtidas na parede anterior do estômago de pacientes submetidos a cirurgia pela técnica da VGP ou da SMAVTP; a probabilidade calculada refere-se ao Teste de Tukey entre Técnicas

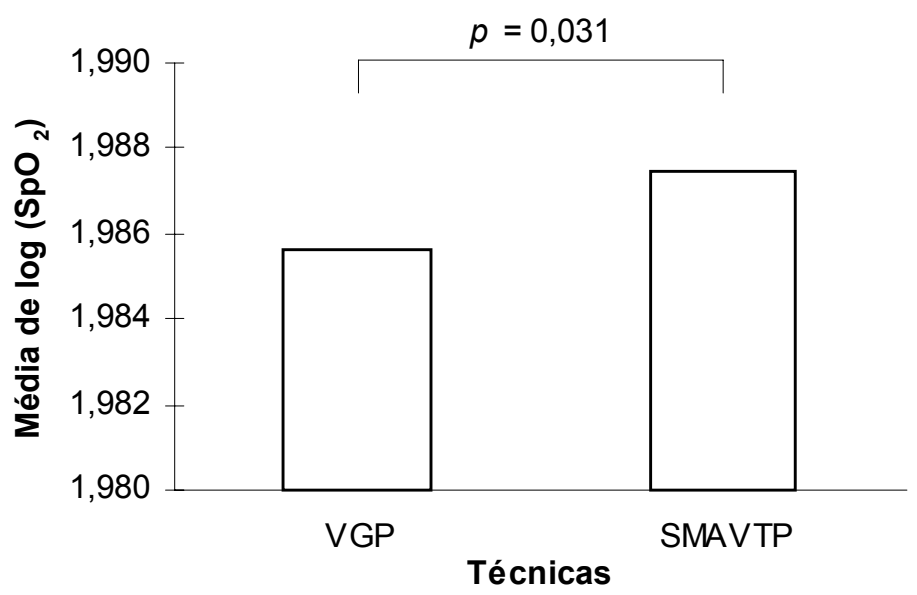

$\left(\mathrm{SpO}_{2}\right)$, respectivamente, entre os seis pontos, entre os três tempos, e entre as duas técnicas, quando consideradas todas as 360 medições de oximetria.

OTeste de Tukey (Gráfico I) foi realizado entre os valores médios de $\log \left(\mathrm{SpO}_{2}\right)$ obtidos nos seis pontos, revelando, entretanto, que apenas os valores medidos nos pontos I (fundo gástrico) e 2 (curvatura menor proximal) foram significantemente menores que em 3 (curvatura menor distal) e 5 (curvatura maior distal), sendo que a redução no ponto I era também estatisticamente significante em relação ao ponto 6 (antro).

Pelo Teste de Tukey (Gráfico 2) também houve diminuição significante na média de log $\left(\mathrm{SpO}_{2}\right)$ pós-procedimento somente quando comparada à do instante inicial. Finalmente, 0 Gráfico 3 permite notar que o Grupo VGP apresentou médias mais baixas que $\circ$ Grupo SMAVTP, tendo o Teste de Tukey mostrado que essa redução era estatisticamente significante $(p=0,031)$.

Cerca de $98,3 \%$ das medidas de $\mathrm{SpO}_{2}$ do presente estudo encontravam-se entre $90 \%$ e $100 \%$. Em cada um dos Grupos pesquisados houve três medidas de oximetria inferiores à saturação de $90 \%$, mas superiores a $80 \%$, sendo que todas estas ocorreram nos pontos correspondentes a o fundo gástrico e curvatura menor proximal.

\section{Discussão}

Atualmente, a cirurgia eletiva da doença ulcerosa tem sido indicada apenas quando há intratabilidade clínica ou quando há falha na erradicação de Helicobacter pylori. ${ }^{9}$ Entre várias técnicas operatórias descritas, temos a VGP eaSMAVTP, as quais parecem ter equivalência em relação às taxas de morbidade e de recorrência ulcerosa pós-operatória, apesar de haver poucos estudos clínicos randomizados comparativos entre elas ${ }^{2,6}$.

Com base em estudo prévio que sugeria comprometimento do esvaziamento gástrico fisiológico para alimentos sólidos após a VGP, sendo que o mesmo não ocorria após a SMAVTP, postulamos a influência da hipóxia, decorrente da desvascularização gástrica nesse achado ${ }^{7}$. Neste estudo, então, procuramos evidenciar diferenças entre a VGP e a SMAVTP, estritamente em relação ao comprometimento da irrigação sanguínea da parede gástrica anterior. Para tanto, utilizamos o método da oximetria de pulso tal como descrito por outros autores ${ }^{10,11,12}$.

O método da oximetria de pulso permitiu apenas 0 acompanhamento intra-operatório das mudanças de saturação de oxigênio da hemoglobina subseqüentes à execução de cada técnica. As medidas foram obtidas antese logo após a ligadura dos vasos gástricos curtos e depois da desnervação da curvatura gástrica menor, de tal forma que cada paciente foi, também, controle dele mesmo.

Nossos resultados mostraram redução estatisticamente significante dos logaritmos da $\mathrm{SpO}_{2}$ na técnica da VGP, quando comparada com a SMAVTP (Gráfico 3), nos pontos correspondentes ao fundo gástrico e à curvatura menor proximal, quando comparados com o corpo gástrico distal (Gráfico I), e no tempo pós-procedimento, quando comparado com o inicial (Gráfico 2).

Estudos anteriores sugerem que a acurácia do método só está comprometida em saturações inferiores a $80 \%$, ${ }^{13,14}$ fato que não ocor- 
reu neste estudo. A confiabilidade do método da oximetria de pulso justifica-se, pois, em tecidos metabolicamente muito ativos, a redução do fluxo sanguíneo tecidual acompanha-se de diminuição da oximetria de pulso, no local da isquemia, devido a maior extração de oxigênio tecidual ${ }^{15,16}$. Esse método mostrou-se fidedigno em nosso estudo, pois nas áreas em que se manteve a vascularização intacta não houve alteração de saturação de oxigênio.

Somente um estudo comparou as duas técnicas quanto à desvascularização gástrica, por meio de arteriografia post-mortem, tendo detectado uma faixa avascular, ao longo da curvatura menor, mais extensa depois de VGP do que após SMAVTP17, entretanto, a diferença não pôde ser avaliada do ponto de vista estatístico, pois os dados eram apenas qualitativos.

Fundoplicatura, ${ }^{18,19}$ mobilização do fundo gástrico com ligadura de vasos gástricos curtos $^{20}$ e esplenectomia, ${ }^{21,22}$ têm sido também incriminadas na ocorrência de isquemia e de necrose do estômago, quando associadas à VGP. No presente estudo, associou-se fundoplicatura rotineiramente tanto à VGP quanto à SMAVTP, com o intuito de prevenir refluxo gastroesofágico conseqüenteà dissecção periesofagiana.

A ligadura de três vasos gástricos curtos era uniformemente realizada a fim de se obter uma fundoplicatura frouxa. O Gráfico 2 mostra que não houve diferença significante entre tempo inicial e pré-procedimento, ou seja, decorrente da ligadura de tais vasos; porém, seu emprego deveria ser criterioso na associação à VGP ou à SMAVTP, pois a redução do $\log \left(\mathrm{SpO}_{2}\right)$ só é significante quando se comparam medidas feitas depois da desnervação das células parietais àquelas obtidas antes da liberação do fundo gástrico pela ligadura dos vasos gástricos curtos (Gráfico 2). Uma constatação importante deste estudo é que a liberação do fundo gástrico, para realização de fundoplicatura, pode acentuar a hipóxia no fundo gástrico e na curvatura menor proximal, quando associada à VGP ou à SMAVTP.

Para explicar o distúrbio no esvaziamento gástrico após a VGP ${ }^{7}$, sugerimos que a desvascularização do estômago possa afetar a função do marcapasso gástrico. Este último, por sua vez, trata-se de um grupo de células conhecidas como células de Cajal ${ }^{23}$, que está localizado na porção média do corpo gástrico, ao longo da curvatura maior, $5 \mathrm{a} 7 \mathrm{~cm}$ aboral à cárdia, e que gera mudança cíclica no potencial, chamada de potencial determinador de freqüência (pacesetter potential) ou ritmo elétrico básico, cuja freqüência é de 3 a 4 ciclos por minuto ${ }^{24,25}$.

Estudos de esvaziamento gástrico para refeição sólida, medido pelo método radioisotópico, têm mostrado a ocorrência de retardo entre 7 a 240 dias após a VGP, sendo que a normalização ocorre até os 6 meses de pósoperatório 5,26,27.

NaSMAVTP, toda a parede gástrica posterior é desnervada pela secção do tronco vagal posterior, mas a motilidade antral deveria permanecer normal, porque se preserva a transmissão de impulsos ao longo do nervo de Latarjet ${ }^{28}$ que, tendo alcançado a túnica muscular antral anterior, são conduzidos à parede antral posterior por arcos vago-vagais intramurais $^{29}$, mantendo inalterado o tempo de esvaziamento para sólidos e líquidos ${ }^{30}$.

Todavia, também foram notados sintomas de retardo do esvaziamento gástrico após a SMAVTP em I,7\% dos pacientes operados ${ }^{31}$, com normalização depois de um ano ${ }^{28}$, mas com ligeiro aumento da freqüência de estase gástrica em relação à VGP32. Apenas um estudo publicado comparou o esvaziamento gástrico de refeição sólida, pelo método radioisotópico, tendo detectado retardo significante após ambos os procedimentos até o sexto mês pós-operatório

Embora alguns acreditem que a VGP não cause distúrbios no marcapasso gástrico ${ }^{25}$, estudos fisiológicos prévios mostraram redução do tempo médio de atividade mioelétrica antral regular e diminuição da amplitude média da onda lenta depois de VGP ${ }^{33}$, com aparecimento ocasional de marcapassos ectópicos, rápidose múltiplos, no estômago distal que, no entanto, não eram suficientes para modificar a motilidade nem o esvaziamento gástrico ${ }^{34}$. Entretanto, taquigastria e bradigastria, associadas a aumento do tempo de esvaziamento gástrico, foram observados em pacientes que apresentavam retardo do esvaziamento mesmo antes da VGP, e foram atribuídas à isquemia das túnicas musculares, causada pela desvascularização gástrica ${ }^{8}$.

A hipótese da participação da isquemia na disfunção do marcapasso gástrico era apoiada por estudo experimental no intestino delgado, no qual notou-se que a hipóxia interferia no marcapasso duodenal, resultando em anomalias na atividade elétrica, na freqüência das ondas lentas e em diminuição da freqüência das contrações intestinais ${ }^{35}$. Por outro lado, a observação clínica de dois casos de gastroparesia permitiu supor que alterações de atividade mioelétrica e de esvaziamento gástrico para sólidos, conseqüentes a isquemia mesentérica crônica, possam ter sido revertidas pela revascularização arteria $^{36}$, confirmando a hipótese enunciada. Esta última também explicaria o esvaziamento pós-operatório significantemente melhor, após a SMAVTP, observado em pacientes operados por úlcera duodenal, quando comparado com a VGP, visto que esta técnica poderia causar maior grau de isquemia da parede gástrica e, portanto, do marcapasso do estômago ${ }^{7}$.

No presente estudo, supomos que 0 ponto 4 , na curvatura gástrica maior proximal, represente a região gástrica próxima de onde se localiza o marcapasso natural, e que alterações oximétricas nele se traduzam por mudanças na oferta de oxigênio às suas células, do qual são dependentes. Assim, observamos que a oximetria média, na região do marcapasso, foi de $95,7 \%$ (mediana de $96,5 \%$ ) depois de VGP, e de $97,2 \%$ (mediana de $97,5 \%$ ) depois de SMAVTP, diferença essa que poderia explicar a aparente superioridade da SMAVTP sobre a VGP. Todavia, não houve diferença estatística da oximetria nessa região em relação ao restante do estômago quando se usou a Análise de Variância. Dessa forma, não pudemos confirmar a ocorrência de hipóxia ao nível do marcapasso gástrico, ainda que houvesse indício desse fenômeno. Amostra de tamanho maior poderia, eventualmente, confirmar a hipótese.

\section{Conclusão}

A Vagotomia Gástrica Proximal, quando associada à ligadura dos vasos gástricos curtos, produz alterações isquêmicas agudas da parede gástrica, detectadas por oximetria de pulso, na região da curvatura menor proximal e do fundo gástrico, mais intensas que a Seromiotomia Anterior com Vagotomia Troncular Posterior. A região do marcapasso gástrico não sofre hipóxia imediatamente após os procedimentos operatórios. 


\section{Agradecimentos}

Aos Professores Dr. Neil Ferreira Novo e Dra. Yara Juliano da Disciplina de Bioestatística da UNIFESP - EPM.

\section{SUMMARY}

Oximetry of stomach: comparative STUDY BETWEEN PROXIMAL GASTRIC VAGOTOMY AND ANTERIOR LESSER CURVE SEROMYOTOMY WITH POSTERIOR TRUNCAL VAGOTOMY IN CHRONIC DUODENAL ULCER

Delayed gastric emptying of solid food is greater after proximal gastric vagotomy (PGV) than after anterior lesser curve seromyotomy with posterior truncal vagotomy (ASPTV) and may be due to the hypoxia in the gastric wall and mainly in the gastric pacemaker.

PURPOSE. To verify if operative devascularization of the lesser curve and the gastric fundus could affect the entire stomach and particularly the pacemaker area.

Methods. Measures of intraoperative oxyhemoglobin saturation $\left(\mathrm{SpO}_{2}\right)$ were taken by pulse oximetry on the anterior gastric wall in 20 patients with chronic duodenal ulcer, randomly allocated in two groups of 10 individuals for surgical treatment, by PGV or ASPTV.

REsULTS. Measurements, before partial fundoplication, showed that the area of the proximal lesser curve and the gastric fundus had a significant decrease in the $\mathrm{SpO}_{2}$ when compared to the gastric corpus $(p<0,05)$; the $\mathrm{SpO}_{2}$ was not reduced significantly by the procedures on the pacemaker area; the ligature of short gastric vessels, in association with PGV or ASPTV, reduced significantly the SpO $(p<0,05)$; and, PGV resulted in a $\mathrm{SpO}$, significantly lower than in ASPTV $(p<0,05)$.

CONCLUSION. When PGV is associated with the ligature of the short gastric vessels, it causes acute ischemic changes in the proximal lesser gastric curve and in the fundus more intensively than ASPTV. The gastric pacemaker area does not present hypoxia immediately after operative procedures. [Rev Assoc Med Bras 2002; 48(4): 323-8]

KeY Words: Stomach. Duodenal Ulcer. Proximal Gastric Vagotomy. Oximetry. Pacemaker.

\section{REFERÊNCIAS}

I. Taylor TV, Gunn AA, MacLeod DAD, MacLennan I. Anterior lesser curve seromyotomy and posterior truncal vagotomy in the treatment of chronic duodenal ulcer. Lancet 1982; 2: 846-9.

2. Oostvogel HJM, Van Vroonhoven TJMV. Anterior lesser curve seromyotomy with posterior truncal vagotomy versus proximal gastric vagotomy. Br J Surg 1 988; 75: I 2 I-4.

3. Amdrup E, Andersen D, Jensen HE. Parietal cell (highly selective or proximal gastric) vagotomy for peptic ulcer disease. World Surg 1977; I: 19-25.

4. Howlett PJ, Sheiner HJ, Barber DC. Gastric emptying in control subjects and patients with duodenal ulcer before and after vagotomy Gut 1976; 17: 542-50.

5. Faxén A, Alpsten M, Cederblad Å, Kewenter], Rossander $L$. The effect of parietal cell vagotomy and selective vagotomy with pyloroplasty on gastric emptying of a solid meal: a prospective randomized study. Scand J Gastroenterol 1978; 13: 727-33.

6. Walia HS, Abd El-Karim H. Anterior lesser curve seromyotomy with posterior truncal vagotomy versus proximal gastric vagotomy: results of a prospective randomized trial 3-8 years after surgery. World J Surg 1994; 18:758-63.

7. Martinez JC. Estudo comparativo do esvaziamento gástrico entre vagotomia gástrica proximal e seromiotomia da pequena curvatura anterior com vagotomia troncular posterior em doentes submetidos a tratamento eletivo da úlcera duodenal (tese). São Paulo: Escola Paulista de Medicina; 1993.

8. Bortolotti M, Labo G, Serantoni C, Ciani P. Effect of highly selective vagotomy on gastric motor activity of duodenal ulcer patients. Digestion 1978; 17:108-20.

9. Trus TL, Hunter JG. Minimally invasive surgery of the esophagus and stomach. Am J Surg 1997; 173:242-55

10. Denobile J, Guzzetta P, Patterson K. Pulse oximetry as a means of assessing bowel viability. J Surg Res 1990; 48:21-3.

II. Katz Y, Shoshani G. Intraoperative assessment of blood flow to strangulated stomach by pulse oximetry. J Pediatr Surg 1992; 27:509-10.

12. Salo JA, Perhoniemi VJ, Heikkinen LO, Verkkala KA, Järvinen AAJ. Pulse oximetry for the assessment of gastric tube circulation in esophageal replacements. Am J Surg 1992; 163:446-7.

13. Cahan C, Decker MJ, Hoekje PL, Strohl KP. Agreement between noninvasive oximetric values for oxygen saturation. Chest 1990; 97:8I4-9.

14. Webb RK, Ralston AC, Runciman WB. Potential errors in pulse oximetry: (II) effects of changes in saturation and signal quality. Anaesthesia 1991; 46:207-12.

15. SeveringhausJW, Spellman MJJr. Pulse oximeter failure thresholds in hypotension and vasoconstriction. Anesthesiology 1990; 73:532-7.
16. MacDonald PH, Dinda PK, Beck IT, Mercer $C D$. The use of oximetry in determining intestinal blood flow. Surg Gynecol Obstet 1993; 176:45I-8.

17. Agossou-Voyème AK, Hureau J, Germain M. Arterial vascularization of the operated stomach: highly selective vagotomy, anterior lesser curve seromyotomy, esophageal replacement by transposed stomach after esophagectomy or circular pharyngolaryngectomy. Surg Radiol Anat 1990; 12:247-57.

18. Kennedy T, Magill P, Johnston GW, Parks TG. Proximal gastric vagotomy, fundoplication, and lesser-curve necrosis. Br Med J 1979 I: $1455-6$

19. Couinaud C. Nécrose ischémique de la petite courbure de l'estomac après vagotomie suprasélective: a propos de 2 cas personnels et de 43 observations publiées dans la littérature. Chir (Paris) 1983; 1 20:77-83.

20. Ackermann C, Müller C, Harder F. Necrosis of intraabdominal esophagus and proximal third of the stomach after proximal gastric vagotomy and fundoplication. World J Surg 1990; 14:133-4.

2I. Kobayasi S, Mendes EF. Irrigação da pequena curvatura após vagotomia gástrica proximal: avaliação pelo método da fluoresceína e estudo histopatológico. Rev Hosp Clin Fac Med Univ São Paulo 1983; 38: I I 5-20.

22. Martinez CAR. Estudo da vascularização do estômago de cães submetidos à vagotomia gástrica proximal isolada ou associada à esplenectomia (dissertação). São Paulo: Escola Paulista de Medicina; 1988

23. Hagger R, Finlayson C, Jeffrey I, Kumar D. Role of the interstitial cells of Cajal in the control of gut motility. Br J Surg 1997; 84:445-50.

24. Weber Jr J, Kohatsu S. Pacemaker localization and electrical conduction patterns in the canine stomach. Gastroenterology 1970; 59:717-26.

25. Hinder RA, Kelly KA. Human gastric pacesetter potential: site of origin, spread, and response to gastric transection and proximal gastric vagotomy. Am J Surg 1 977; 1 33:29-33.

26. Sheiner HJ, Duthie HL, Barber DC, Howlett PJ. Proceedings: the effect of highly selective vagotomy and truncal vagotomy with pyloroplasty on gastric emptying of solid food in man. Br J Surg 1975; 62:163-4.

27. Lopasso FP, Meneguetti JC, Mello JB, GamaRodrigues], Raia AA. Estudo do esvaziamento gástrico em ulcerosos duodenais antes e após vagotomia gástrica proximal. Rev Assoc Med Bras 1983; 29:10-3.

28. Taylor TV, Lythgoe JP, McFarland JB. Anterior lesser curve seromyotomy and posterior truncal vagotomy versus truncal vagotomy and pyloroplasty in the treatment of chronic duodenal ulcer. Br J Surg 1990; 77:1007-9.

29. Daniel EE, Sarna SK. Distribution of excitatory vagal fibers in canine gastric wall to control motility. Gastroenterology 1976; 7|:608-|3. 
30. Taylor TV, Holt S, Heading RC. Gastric emptying after anterior lesser curve seromyotomy and posterior truncal vagotomy. Br JSurg 1985; 72: 620-2.

3I. Taylor TV, Gunn AA, MacLeod DAD. Mortality and morbidity after anterior lesser curve seromyotomy with posterior truncal vagotomy for duodenal ulcer. $\mathrm{Br}$ J Surg 1985; 72:950-I.

32. Johnston D, Blackett RL. A new look at selective vagotomies. Am J Surg 1988; 156:4 I6-27.
33. Stoddard CJ, Waterfall WE, Brown BH, Duthie $\mathrm{HL}$. The effects of varying the extent of the vagotomy on the myoelectrical and motor activity of the stomach. Gut 1973; 14:657-64.

34. Wilbur BG, KellyKA. Effect of proximal gastric, complete gastric, and truncal vagotomy on canine gastric electric activity, motility and emptying. Ann Surg 1973; 178: 295-303.

35. Szurszewski J, Steggerda FR. The effect of hypoxia on the electrical slow wave of the canine small intestine. Am J Dig Dis 1968; 1 3:168-77.
36. Liberski SM, Koch KL, Atnip RG, Stern RM. Ischemic gastroparesis: resolution after revascularization. Gastroenterology 1990; 99:252-7.

Artigo recebido: 02/08/2001

Aceito para publicação: 30/10/2001

\section{IMAGEM EM MEDICINA}

Participe da nova seção da Ramb. É um espaço aberto ao leitor, que poderá enviar material de interesse educativo como fotos, ilustrações e exames, acrescido de três linhas explicativas contendo ainda nome do autor e serviço onde foi realizado.

O material poderá ser enviado para a

Rua São Carlos do Pinhal, 324 - Cep 01333-903

S. Paulo - SP - E-mail: ramb@amb.org.br 